\title{
Sprachförderung in Kindertagesstätten
}

Tanja Jungmann

Inhaltsverzeichnis

14.1 Einleitung - 310

14.2 Sprachförderung in den Bildungsplänen der Länder - 311

14.2.1 Diagnostik sprachlicher Kompetenzen - 311

14.2.2 Sprachbildung vs. Sprachförderung - 313

14.2.3 Umgang mit mangelnder Sprachbeherrschung - 313

14.3 Additive vs. alltagsintegrierte Sprachförderung - 314

14.3.1 Additive Sprachförderung - 314

14.3.2 Alltagsintegrierte Sprachförderung - 317

14.4 Implikationen für die Praxis - 321

14.4.1 Erfassung sprachförderrelevanter Interaktionen - 322

14.4.2 Erforderliche Kompetenzen aufseiten der Fachkräfte - 323

14.5 Zusammenfassung - 325

14.6 Weiterführende Literatur - 325

Literatur - 326 
Die Effekte von sprachlicher Bildung und Sprachförderung hängen davon $a b$, wie kompetent pädagogische Fachkräfte den Alltag adaptiv (angepasst an die sprachlichen Kompetenzen der Kindergruppe und einzelner Kinder) sprachförderlich zu gestalten vermögen.

\subsection{Einleitung}

Wie können pädagogische Prozesse so gestaltet werden, dass das vorhandene Wissen für die Sprachförderung in pädagogischen Interaktionen, aber auch in Kooperation mit den Eltern umgesetzt werden kann? Einen Ausgangspunkt zur Beantwortung dieser Frage bieten die 5 Qualitätsdimensionen in Anlehnung an Tietze et al. (2005).

Mit der Kontextqualität sind Impulse gemeint, die aus dem Umfeld der Kindertageseinrichtung, dem Stadtteil mit seinen sozialen Lagen und Angeboten, kommen, die auch von der Einrichtung genutzt werden können.

Die Orientierungsqualität in der Kindertageseinrichtung bezieht sich zum einen darauf, wie klar und differenziert die pädagogischen Ziele einer Einrichtung konzeptionell formuliert sind, zum anderen sind die Orientierungen der Fachkräfte durch deren Menschenbilder, insbesondere das Bild vom Kind, und ihre Haltungen, die das pädagogische Handeln im Sinne eines generativen Prinzips leiten, begründet. Hier bieten die Bildungspläne der Länder allgemeine Anhaltspunkte, wie die Sprachförderung in der Kindertageseinrichtung gestaltet werden sollte ( Abschn. 14.2). Spezifischere Hinweise zur Erreichung der Ziele in der sprachlichen Kompetenzentwicklung der Kinder enthalten allerdings die Sprachförderprogramme ( Abschn. 14.3).

In der sächlichen, räumlichen und personellen Ausstattung zeigt sich die Strukturqualität einer Einrichtung. Sie wird über Gruppengrößen und die FachkraftKind-Relation bestimmt, bemisst sich aber auch nach der Vorbereitungszeit, die für die direkte Arbeit mit den Kindern zur Verfügung steht, und dem Ausbildungsniveau der pädagogischen Fachkräfte. Internationale Längsschnittstudien belegen den Einfluss der strukturellen Ressourcen von Kindertageseinrichtungen auf die Qualität der pädagogischen Prozesse (z. B. ECCE 1999). In der Untersuchung von Tietze et al. (2005) ließ sich beinahe die Hälfte der Varianz der Prozessqualität zwischen den Kindergartengruppen auf Unterschiede in der Struktur- und Orientierungsqualität zurückführen. In diesem Zusammenhang werden immer wieder die zu schlechte Ausstattung der Kindertageseinrichtungen mit Personal sowie Ausbildungsstandards, die weit hinter dem Niveau anderer europäischer Länder zurückbleiben, moniert (Strehmel 2010).

Die Prozessqualität bezieht sich schließlich auf die FachkraftKind-Interaktionen, aber auch auf die Peer-Interaktionen und die Kommunikation innerhalb des pädagogischen Teams. Die Qualität dieser Prozesse zeigt sich in der Bildungsatmosphäre und in den adaptiven Lern- und Erfahrungsmöglichkeiten des Kindes (Beckerle und Mackowiak 2019). Diese wirkt sich unmittelbar auf die Entwicklungsprozesse der Kinder aus. Um eine optimale Prozessqualität zu erreichen, sind eine ausgeprägte, durch ein hohes Maß an Reflexion gekennzeichnete Orientierungsqualität, eine gute und durch ausreichende Ressourcen getragene Strukturqualität sowie ein professionelles Management notwendige Voraussetzungen.

$\mathrm{Zu}$ den Organisations- und Managementqualitäten gehören, neben der fachlichen Leitung, die Bewirtschaftung und Personalführung, die Kooperation mit den Eltern, die Öffentlichkeitsarbeit und die Kontakte mit dem Träger, den Behörden usw. Ein 
gutes Management gilt als Voraussetzung für die Leistungsfähigkeit des Personals in der Gestaltung der pädagogischen Prozesse mit den Kindern (Strehmel 2010).

Im Folgenden werden zunächst entlang der Dimension Orientierungsqualität Gemeinsamkeiten und Unterschiede in den Bildungsplänen der Länder zur Sprachbildung und -förderung skizziert. Anschließend wird am Beispiel spezifischer Sprachförderkonzepte auf die konkrete Gestaltung der Prozessqualität und die sich daraus ergebenden Anforderungen für die pädagogischen Fachkräfte eingegangen.

\subsection{Sprachförderung in den Bildungsplänen der Länder}

Die Ergebnisse nationaler und internationaler Schulleistungsvergleiche (OECD 2001, 2006, 2011) wiesen auf einen engen Zusammenhang zwischen den sprachlichen Kompetenzen eines Kindes und seinem Schulerfolg hin. Damit rückte das Thema „sprachliche Bildung" im Allgemeinen und „Sprachförderung“ im Besonderen in allen Bundesländern verstärkt in den bildungspolitischen Fokus. Dies fand seinen Niederschlag in den Orientierungsund Bildungsplänen, die sich allerdings in ihren Aussagen zur Diagnostik von Sprachkompetenz, zur Sprachbildung und zur Sprachförderung zum Teil deutlich unterscheiden, aber auch Gemeinsamkeiten aufweisen (Jungmann und Albers 2013).

\subsubsection{Diagnostik sprachlicher Kompetenzen}

Insgesamt ist $\mathrm{zu}$ konstatieren, dass sich in den Bildungsplänen der Länder nur sehr wenige Hinweise zum konkreten diagnostischen Vorgehen in Kindertageseinrichtungen finden lassen. Dies ist damit zu erklären, dass die Praxis der Erhebung des Sprachstands in gesetzlichen Vorgaben zumeist außerhalb der Bildungspläne konkretisiert wird.

Bayern betont als einziges Bundesland die Bedeutung der Früherkennung von Entwicklungsrisiken und die damit verbundenen Qualifikationsanforderungen an frühpädagogische Fachkräfte. Diese sollen die unterschiedlichen Verfahren zur Erfassung und Dokumentation sprachlicher Fähigkeiten beherrschen und im Sinne der Sprachförderung einsetzen können. Zur Identifizierung von Risikokindern und zur Planung und Abstimmung der Fördermaßnahmen wird zudem zur intensiven Kooperation mit Fachdiensten geraten (Bayerisches Staatsministerium für Arbeit und Sozialordnung, Familie und Frauen 2016).

Auch die Fachkräfte in schleswigholsteinischen Kindertageseinrichtungen sind dazu angehalten, sonderpädagogische Lehrkräfte mit den Schwerpunkten Sprache und Sprechen sowie Fachpersonen aus den Bereichen Logopädie und Sprachtherapie hinzuziehen, wenn es um die Abgrenzung zwischen Sprachförderbedarfen und Sprachstörungen geht (Ministerium für Soziales, Gesundheit, Familie und Gleichstellung des Landes Schleswig-Holstein 2012).

In den Bildungsplänen der Länder Schleswig-Holstein, Bayern und Niedersachsen (Niedersächsisches Kultusministerium 2005) wird für die systematische Entwicklungsbegleitung sprachlicher Kompetenzen die Einführung der Beobachtungsbögen „Sprachverhalten und Interesse an Sprache bei Migrantenkindern in Kindertageseinrichtungen" (SISMIK; Ulich und Mayr 2003) und ,Sprachentwicklung und Literacy bei deutschsprachig aufwachsenden Kindern" (SELDAK; Ulich und Mayr 2006) empfohlen. Während der SISMIK für die systematische Erfassung und Dokumentation der Sprach- und Literacy-Entwicklung bei mehrsprachig aufwachsenden Kindern im 
Alter von 3;5 Jahren bis zum Schuleintritt konzipiert wurde, richtet der SELDAK den Aufmerksamkeitsfokus der pädagogischen Fachkraft auf Kinder mit der Erstsprache Deutsch. Ausführlichere Informationen zum SELDAK sind in dem Beispiel: Beobachtungsbogen SELDAK (Ulich und Mayr 2006) zu finden.

\section{Beispiel: Beobachtungsbogen SELDAK} (Ulich und Mayr 2006)

Der Beobachtungsbogen SELDAK ermöglicht eine strukturierte Einschätzung der kindlichen Sprach- und Literacy-Entwicklung bei Kindern im Alter von 4 Jahren bis zum Schuleintritt im pädagogischen Alltag. Theoretische Grundlagen sind Forschungsergebnisse über die frühen literalen Kompetenzen, das Interesse an sprachbezogenen Aktivitäten und den Erwerb von Morphologie und Syntax in dieser Altersspanne.

Der Beobachtungsbogen umfasst die beiden folgenden Teile:

1. Sprachrelevante Situationen: Aktivität und Kompetenzen:

- Gesprächsrunden und Gruppendiskussionen

- Bilderbuchbetrachtung als pädagogisches Angebot in der Kleingruppe oder in der Zweiersituation (Bezugsperson - Kind)

- Vorlesen/Erzählen als pädagogisches Angebot in der Kleingruppe

- Selbstständiger Umgang mit Bilderbüchern (vom Kind ausgehend)

- Kinder als Erzähler (in der Gruppe, in Zweiersituationen); Kinder erzählen eigene Erlebnisse, eine Geschichte, ein Märchen

- Kommunikatives Verhalten in Gesprächssituationen

- Lausch- und Reimspiele

- Schreiben/Schrift
2. Sprachliche Kompetenzen im engeren Sinne:

- Verstehen von Handlungsaufträgen/ Aufforderungen

- Wortschatz

- Grammatik: Morphologie, Syntax

- Dialekt - Hochdeutsch

- Sprechweise

- Nachsprechen von Sätzen

Der Beobachtungsbogen wurde an ca. 2500 Kindern empirisch untersucht und seine Reliabilität und Validität bestätigt. Er erlaubt es, unter Praxisbedingungen mit überschaubarem Aufwand breite und zuverlässige Einblicke in die sprachliche Entwicklung von Kindern zu gewinnen. Darüber hinaus wirkt er als Qualifizierungsmaßnahme für Fachkräfte bezogen auf die Förderung von Sprache und Literacy in Kindertageseinrichtungen (Mayr und Ulich 2010).

Das Land Brandenburg verfolgt ebenfalls die regelmäßige und differenzierte Beobachtung von Kindern mit dem Ziel der Erfassung von Stärken und Vorlieben auf sprachlicher Ebene (Ministerium für Bildung, Jugend und Sport, Land Brandenburg 2006). Zusätzlich zur Beobachtung der sprachlichen Entwicklung wird im Hamburger Bildungsplan eine Kombination von standardisiertem Sprachscreening mit dem „Hamburger Verfahren zur Analyse des Sprachstands Fünfjähriger" (HAVAS 5; Reich und Roth 2004) und prozessorientierter Begleitung der sprachlichen Fähigkeiten präferiert (Behörde für Arbeit, Soziales, Familie und Integration, Hamburg 2012).

Niedersachsen und Brandenburg betonen bei der prozessorientierten Beobachtung und Dokumentation der Sprachkompetenz dagegen die Bedeutung der Kooperation mit der Familie. Die Beobachtungen der frühpädagogischen Fachkraft liefern einerseits Anhaltspunkte für Förderangebote im Alltag der Einrichtung, andererseits stellen sie eine Grundlage für die Zusammenarbeit 
mit den Eltern dar. Niedersachsen betont darüber hinaus die Bedeutung der intensiven Kooperation mit den Eltern mit Migrationshintergrund bei der Identifizierung der sprachlichen Kompetenzen in der Herkunftssprache.

\subsubsection{Sprachbildung vs. Sprachförderung}

Während in den meisten Bildungsplänen die Begriffe ,sprachliche Bildung“ und „Sprachförderung“ synonym verwendet werden, findet sich eine Differenzierung im Bildungsplan Niedersachsens.

\section{Definition}

Sprachliche Bildung ist demnach grundsätzlicher Bestandteil des Kindergartenalltags und kann durch die Bereitstellung einer anregenden Lernumwelt intensiviert werden. In diesem Zusammenhang erweisen sich Lieder, Reime und Singspiele verbunden mit Bewegung als besonders wirkungsvoll. Kinder erwerben ganz beiläufig die prosodischen, phonetisch-phonologischen, semantisch-lexikalischen und morphologisch-syntaktischen Elemente der Sprache und begegnen der Sprache in einer Weise, mit der sie einen Sinn verknüpfen. Sprachliche Bildung wird als durchgängiges Prinzip der pädagogischen Arbeit in der Kindertageseinrichtung verstanden. Zudem wird Sprache nicht als isoliertes Phänomen betrachtet, sondern in der Verknüpfung mit anderen Entwicklungsbereichen ( Abschn. 14.3.2).

Sprachförderung ist dagegen eine spezifische, gezielte Unterstützung der sprachlichen Fähigkeiten von Kindern, bei denen ein Förderbedarf identifiziert wurde, charakterisiert. Die Sprachförderung kann in Kleingruppen oder alltagsintegriert stattfinden. Die frühpädagogischen
Fachkräfte setzen reflektiert Korrekturund Modellierungstechniken in einem für die Kinder bedeutungsvollen Rahmen ein $(\triangleright$ Abschn. 14.3.1).

Die Einbindung der sprachlichen und frühen literalen Förderung in den Alltag der Kindertageseinrichtung wird auch in den Bildungsplänen Thüringens (Ministerium für Bildung, Jugend und Sport, Thüringen 2015) und Mecklenburg-Vorpommerns (Ministerium für Bildung, Wissenschaft und Kultur Mecklenburg-Vorpommern 2011) betont. Demnach sind sprachliche und schriftsprachliche Bildungsprozesse nicht auf eine dafür vorgesehene Zeitspanne im Tagesablauf $\mathrm{zu}$ beschränken, sondern beziehen alle Situationen mit ein, in denen sich Kinder bilden. Die Fachkräfte schaffen dafür die Rahmenbedingungen und halten Angebote vor, um die sprachliche und die frühe literale Bildung der Kinder anzuregen und nachhaltig $\mathrm{zu}$ unterstützen. Zur Grundausstattung einer Kindertageseinrichtung gehören - dem Bildungsplan Schleswig-Holsteins zufolge - gut ausgestattete Bibliotheken mit Bilderbüchern, Lese-, Hör- und Schreibecken.

\subsubsection{Umgang mit mangelnder Sprachbeherrschung}

Es wird davon ausgegangen, dass institutionelle Bildungseinrichtungen wie die Kindertageseinrichtung und die Schule eine geringe sprachliche Anregung in der Familie zu kompensieren vermögen.

Bremens Bildungsplan enthält explizite Hinweise zum Einsatz der Sprache durch die frühpädagogische Fachkraft in Gesprächen mit einzelnen Kindern und innerhalb der Vielzahl an natürlichen Sprechanlässen während des Tagesablaufs (Senatorin für Soziales, Jugend, Frauen, 
Integration und Sport, Bremen 2012). Dabei kommt dem handlungsbegleitenden Sprechen eine hohe Bedeutung zu, Absichten und Interessen werden im Dialog geklärt, Fragen beantwortet, Konflikte bearbeitet und Planungen gemeinsam diskutiert. Beim Austausch von Ergebnissen werden darüber hinaus narrative und dialogische Kompetenzen unterstützt und erweitert. Die Komplexität und Äußerungslänge der Sprache der Fachkraft richtet sich dabei nach den Kompetenzen der Kinder, um einerseits genügend Anregung zu bieten, andererseits aber nicht zu überfordern.

Der Hinweis auf entsprechende Methoden, z. B. den expliziten Einsatz von Korrektur- und Modellierungstechniken, um sprachliche Zielformen in den Fokus der kindlichen Aufmerksamkeit zu rücken, findet sich u. a. auch im Bildungsplan Schleswig-Holsteins. Dies zielt vorrangig auf die rezeptive Dimension der Sprachentwicklung $a b$, um den Aufbau einer inneren Repräsentation der Zielstruktur zu ermöglichen und Kinder zur Produktion zielsprachlicher Äußerungen zu befähigen. Die Korrektur- und Modellierungstechniken sollten variabel eingesetzt und die Form- und Funktions-Beziehungen der Zielformen in kontrastierender Weise hervorgehoben werden. Die Prägnanz des Einsatzes der expliziten Techniken im Diskurs ist für die Überführung der Zielstruktur in die Spontansprache des Kindes effektiver als die Quantität durch Wiederholung der Äußerung.

Im Unterschied $\mathrm{zu}$ allen anderen Bundesländern sieht der Bildungsplan Sachsen-Anhalts die Beherrschung des Hochdeutschen durch die frühpädagogische Fachkraft als wichtige Voraussetzung für die Unterstützung sprachlicher Fähigkeiten an (Ministerium für Arbeit und Soziales des Landes Sachsen-Anhalt 2014). Zur gezielten und bewussten Unterstützung des
Spracherwerbs sind dem Bildungsplan Niedersachsens entsprechend Kenntnisse über den normalen und auffälligen Erst- und Zweitspracherwerb unerlässlich. In diesem Zusammenhang wird auch die Bedeutung der Peer-Interaktionen im Bildungsplan Bayerns in sehr differenzierter Weise herausgearbeitet. Kinder, die in einer Einrichtung mit einem hohen Anteil von Kindern nicht deutscher Herkunftssprachen aufwachsen, benötigen eine intensivere Förderung in Kleingruppen, Einzelfördersituationen, stärkere Zusammenarbeit mit der Familie sowie eine Öffnung der Einrichtung für die Kooperation mit Semiprofessionellen wie Vorlesepaten und Personen mit einer anderen Herkunftssprache als Deutsch. Im Unterschied $\mathrm{zu}$ allen anderen Bundesländern greift Bayern auch das Aufwachsen in einem ,sozialen Brennpunkt" als eine problematische Entwicklungsbedingung auf. Dies gilt vor allem dann, wenn diese Kinder auch innerhalb der Peergroup nur eingeschränkte Kontaktmöglichkeiten zur Umgebungssprache Deutsch erhalten.

\subsection{Additive vs. alltagsintegrierte Sprachförderung}

Spezifischer als die Bildungspläne formulieren Sprachförderprogramme Ziele im Hinblick auf die Kompetenzentwicklung der Kinder. Nach Lisker (2011) wird dabei zwischen sprachstrukturellen (additiven) und ganzheitlichen (alltagsintegrierten) Sprachförderkonzepten unterschieden.

\subsubsection{Additive Sprachförderung}

Es existiert eine Vielzahl von Programmen zur Förderung der sprachlichen Fähigkeiten im Kindergartenalter (Jampert et al. 
2007), die für die folgenden Zielgruppen konzipiert sind:

- Alle Kinder (universelle Sprachförderung)

- Kinder mit Migrationshintergrund bzw. mehrsprachig aufwachsende Kinder

- Kinder aus bildungsfernen oder sozial schwachen Schichten

- Kinder mit Problemen des Schriftspracherwerbs

- Kinder mit Sprachentwicklungsstörungen (SES)

Bei der Durchführung sprachstruktureller, additiver Programme von Sprache oder Literacy, z. B. „Sprache macht stark“ von Tracy und Lehmke (2009), „Kon Lab" von Penner und Krügel (2007) oder „Hören, lauschen, lernen“ von Küspert und Schneider (2008), werden einzelne oder mehrere Sprachebenen (z. B. Wortschatz, Grammatik, phonologische Bewusstheit) systematisch nach einem festgelegten zeitlichen Ablaufplan mit vorgegebenem Material gefördert. Mit den Programmen zur Sprachförderung geht in der Regel auch die Fortbildung der pädagogischen Fachkräfte einher.

Auffällig viele Programme entfallen auf die Altersgruppe der 5-Jährigen, erst in den vergangenen Jahren wurden auch die 0- bis 4-Jährigen stärker in den Blick genommen (Winner 2007). Schrey-Dern (2006) kritisiert, dass die Programme, die sich an alle Kinder richten, auch durchaus Kinder mit SES meinen, d. h., hier werde vermittelt, dass eine Sprachförderung ausreichend sei, um die vorliegende Sprachstörung zu beheben. Liegen aber spezifische Störungen des Spracherwerbs oder allgemeine Entwicklungsstörungen vor, ist eine zusätzliche Sprachtherapie unverzichtbar (vgl. hierzu Kap. 15). Nichtsdestotrotz sollten diese Kinder in der Interaktion mit den Fachkräften und anderen Kindern sprachliche und literale Anregungen erhalten. Diese sind dann allerdings nicht im präventiven Sinne zu verstehen, sondern als flankierend zur Therapie, die außerhalb der Kindertageseinrichtung stattfindet.

Des Weiteren sind sprachstrukturelle Förderprogramme in den letzten Jahren vermehrt kritisiert worden, weil es ihnen oftmals an einer empirischen Wirksamkeitskontrolle fehlt. Wenn jedoch ihre Effektivität systematisch überprüft wird, ist das Ergebnis vielfach eher ernüchternd, wie die EVAS-Studie (Schöler und Roos 2010) zeigt (für weitere Informationen s. Jungmann 2009; Petermann 2015; Schneider 2018; Schöler und Roos 2010).

\section{EVAS-Studie (Schöler und Roos 2010)}

Im Rahmen der Studie zur Evaluation von Sprachförderung bei Vorschulkindern (EVAS) wurde die Wirksamkeit von 3 vorschulischen Sprachförderkonzepten nach Penner (2003), Tracy (2003) und Kaltenbacher und Klages (2005) im Vergleich zu einer unspezifischen Sprachförderung untersucht (Schöler und Roos 2010). Die spezifische Sprachförderung wurde in Kleingruppen mit 6-10 Kindern über einen Zeitraum von mindestens 6 Monaten mit einer Förderzeit von 4 bis $5 \mathrm{~h}$ pro Woche ausschließlich von entsprechend ausgebildeten Sprachförderkräften durchgeführt.

Die Effektivität der Förderprogramme wurde im Rahmen eines Prätest-Posttest-Designs mit 3 Untersuchungsgruppen evaluiert, die 199 Kinder ohne Förderbedarf, 82 sprachlich auffällige Kinder, die unspezifisch gefördert wurden, sowie 209 Kinder, die an einem der 3 spezifischen Sprachförderprogramme partizipiert haben, umfassten. Der Untersuchungszeitraum erstreckte sich vom Beginn des letzten Kindergartenjahres (2005) bis zum Ende der 2. Klasse (2008). Um den Förderbedarf der Kinder zu diagnostizieren, wurden verschiedene Verfahren eingesetzt: SISMIK (Ulrich und Mayr 2003), Heidelberger Auditives Screening in der Einschulungsuntersuchung (HASE; Brunner und Schöler 2002) und Einschätzungen des Sprachentwicklungsstands durch die pädagogischen Fachkräfte. Zur Erfassung von Veränderungen der sprachlichen Kompetenzen der Kinder über die Zeit wurden vor und nach der Förderung 4 Untertests des Heidelberger Sprachentwicklungstests (HSET; Grimm und Schöler 1991) - Imitation von Sätzen (IS), Verstehen grammatischer Strukturformen (VS), Plural-Singular-Bildung (PS) und Wortfindung (WF) durchgeführt. Weiterhin wurde ein informelles Verfahren zur Wortschatzprüfung aus dem Ravensburger Prüfmaterial (Frank und Grziwotz 2001) eingesetzt. Kontrolliert wurden der Einfluss der nonverbalen Intelligenz der Kinder mithilfe von Coloured 
Progressive Matrices (CPM; Raven 2002) sowie der Einfluss des sozioökonomischen Status (Elternfragebogen).

Nach der Förderphase hatte sich der Leistungsabstand zwischen den förderbedürftigen Gruppen und der Gruppe ohne Förderbedarf nur unwesentlich verringert. Dabei zeigten die unspezifisch geförderten Kinder ähnliche Leistungen wie jene, die nach spezifischen Sprachprogrammen gefördert wurden. Dieses Ergebnis blieb auch in einem Post-hoc-Vergleich zwischen den einzelnen Fördergruppen und der Gruppe ohne spezifische Förderung bestehen, d. h., keines der 3 Programme war den unspezifischen Fördermaßnahmen überlegen.

Eine Ausnahme bilden die Programme zur Förderung der phonologischen Bewusstheit im weiteren und engeren Sinne im letzten Kindergartenjahr wie das Würzburger Trainingsprogramm „Hören, lauschen, lernen" (Küspert und Schneider 2008) und die kindergartenbasierte Förderung „Lobo vom Globo" (Fröhlich et al. 2010), die mit sehr guten Ergebnissen evaluiert wurden (Evaluationsstudien zur Förderung phonologischer Bewusstheit).

\section{Evaluationsstudien zur Förderung phono- logischer Bewusstheit}

Zum Würzburger Trainingsprogramm liegen 3 große kontrollierte Evaluationsstudien mit längsschnittlichem Kontrollgruppendesign und Nachfolgeuntersuchungen vor.

In den ersten beiden Studien aus den Jahren 19911994 (Schneider et al. 1994) sowie 1993-1997 (Schneider et al. 1997) mit anfallenden Stichproben stellten sich der erwartete Trainingseffekt auf die phonologische Bewusstheit und die positive Auswirkung auf das Lesen und Schreiben im Vergleich zur nicht trainierten Kontrollgruppe ein. Dies geschah allerdings nur dann, wenn das Training konsequent und bis zum Ende durchgeführt wurde und die Erzieher/-innen gut supervidiert wurden.

In der 3. Studie wurden erstmals speziell „Risikokinder" im Hinblick auf die spätere Lese- und Rechtschreibentwicklung trainiert (Schneider et al. 2000), die zuvor durch das Bielefelder Screening zur Früherkennung von Lese-Rechtschreibschwierigkeiten (BISC; Jansen et al. 2002) identifiziert worden waren. In dieser Studie wurde auch die Effektivität von 3 Trainingsvarianten (Training der phonologischen Bewusstheit vs. Buchstabe-Laut-Training vs. kombiniertes Training mit $75 \%$ der phonologischen und $60 \%$ der Buchstabe-Laut-Einheiten) miteinander verglichen. Die phonologische Bewusstheit der Risikokinder konnte durch das phonologische und das kombinierte Training sehr erfolgreich gefördert werden. Allerdings schnitt die „unausgelesene" Kontrollgruppe, die größtenteils aus Nichtrisikokindern bestand, sowohl beim Lesen als auch beim Rechtschreiben am besten ab. Hinsichtlich des späteren Lesens und Schreibens profitierte die kombiniert trainierte Risikokindergruppe am meisten. Darüber hinaus liegen zahlreiche Erfahrungsberichte zur Durchführbarkeit des Würzburger Trainingsprogramms vor. Die Rückmeldungen der Fachkräfte waren überwiegend positiv. Allerdings moniert Schmid-Barkow (1999), dass die Auswahl des verwendeten Sprachmaterials wenig kindgerecht erscheine, aus linguistischer Perspektive nicht immer stimmig sei und durch die strikte Festlegung der einzelnen Übungseinheiten in Ablauf und Abfolge hohe Ansprüche an die Disziplin der pädagogischen Fachkräfte und der Kinder stelle. Für eine effektive Durchführung des Gruppentrainings sind sicherlich eine aufmerksame Beobachtung der individuellen Schwierigkeiten einzelner Kinder, Kreativität im Umgang mit solchen Problemen sowie eine regelmäßige Reflexion im pädagogischen Team und Erfahrungsaustausch zwischen den Fachkräften notwendig (Weber et al. 2007).

Die kindergartenbasierte Lobo-Förderung wurde an einer Stichprobe von 191 Kindergartenkindern aus Bremen und Niedersachsen systematisch überprüft (Fröhlich et al. 2008). Die Eltern der Kinder wurden durch Informationsabende und Flyer über die Studie informiert. Während in der einen Hälfte der Einrichtungen das Lobo-Programm durchgeführt wurde, stellte sich die andere Hälfte als Vergleichsgruppe zur Verfügung, d.h., die Kinder nahmen nur an den Testungen teil, erhielten aber keine spezifische Förderung. Vor Beginn der 3-monatigen Trainingsdurchführung fand eine Schulung für die pädagogischen Fachkräfte statt. Um die Ausprägung der phonologischen Bewusstheit zu überprüfen, wurde zu beiden Messzeitpunkten der Test für Phonologische Bewusstheitsfähigkeiten (TPB; Fricke und Schäfer 2011) eingesetzt. In allen 7 Untertests erzielten die Kinder der Fördergruppe deutlichere Leistungszuwächse als die Kinder der Vergleichsgruppe. Weiterhin wiesen sie weniger unterdurchschnittliche Testergebnisse auf.

Die Programmakzeptanz bei den Kindern fiel durchgängig hoch aus: $99 \%$ der Kinder gaben an, dass ihnen die Zeit mit dem Drachen Lobo gefallen habe; $85 \%$ würden gerne noch einmal Aufgaben mit Lobo zusammen bearbeiten. Diese Einschätzung wurde auch von den pädagogischen Fachkräften geteilt. 


\subsubsection{Alltagsintegrierte Sprachförderung}

Elementarpädagogisch orientierte, ganzheitliche Sprachförderkonzepte, zu denen auch die alltagsintegrierte Sprachförderung gehört, stehen in der Tradition des situationsorientierten Ansatzes. Diesem zufolge wird die Sprachkompetenz nicht in punktuellen, zeitlich begrenzten Fördereinheiten erworben, sondern durch Sprechen mit Erwachsenen, Gleichaltrigen, älteren und jüngeren Kindern in Alltagssituationen. Die Sprachförderung knüpft an die aktuellen Bedürfnisse und Interessen der Kinder an, wobei ihr Interesse und ihre Motivation einer Aktivität gehören sollten, in der Sprache Mittel zum Zweck ist.

Im Unterschied zur additiven Sprachförderung werden zwar Rahmenkonzepte vorgegeben, aber keine konkreten Inhalte. Vielmehr ist Sprachförderung durchgängiges Prinzip zur Gestaltung des Alltags in der Kindertagesstätte und erfolgt nicht nur punktuell oder $\mathrm{zu}$ bestimmten Fördereinheiten. Ziel ist es, die sprachlichen Kompetenzen der Kinder von Anfang an umfassend zu fördern, Auffälligkeiten rechtzeitig $\mathrm{zu}$ erkennen und schulvorbereitend $\mathrm{zu}$ wirken, da das sprachliche Interaktionsverhalten in Elternhaus und Kindertagesstätte ein guter Prädiktor der späteren Sprach-, Lese- und Rechtschreibleistungen der Kinder ist.

Im Rahmen des Forschungs- und Entwicklungsprogramms „Bildung durch Sprache und Schrift" (BiSS) werden die in den Bundesländern eingeführten Angebote zur Sprachförderung im Elementarbereich im Hinblick auf ihre Wirksamkeit und Effizienz geprüft und wissenschaftlich weiterentwickelt. Der Qualitätscheck der alltagsintegrierten Förderkonzepte und -instrumente kommt zu dem Schluss, dass entweder nur einzelne oder lediglich informelle Teilziele erreicht werden konnten oder dass keine Aussagen zur Wirksamkeit vorliegen (BiSS-Trägerkonsortium 2020; weitere Informationen unter: $>$ https://bisssprachbildung.de/ueber-biss/).

$\mathrm{Zu}$ ähnlichen Schlussfolgerungen kommen Egert und Hopf (2016) in ihrem narrativen Review zur Wirksamkeit von Sprachförderung in Kindertageseinrichtungen. Sie konstatieren zudem eine eher inkonsistente Forschungslage bei Kindern im Alter von 3 bis 6 Jahren. Bei den unter 3-Jährigen ließen sich aber in 2 Studien positive Effekte von alltagsintegrierter Förderung auf die Sprachleistungen nachweisen.

Exemplarisch für das alltagsintegrierte Vorgehen werden im Folgenden das „Heidelberger Interaktionstraining für pädagogisches Fachpersonal zur alltagsintegrierten Sprachbildung und Sprachförderung" (HIT; Buschmann et al. 2010) sowie das „KOMPASS-Projekt“, ein Professionalisierungsangebot für pädagogische Fachkräfte u. a. im Bildungsbereich Sprache/Literacy (Jungmann et al. 2015), kurz beschrieben und Evaluationsergebnisse berichtet.

\subsubsection{Heidelberger Interaktionstraining für pädagogisches Fachpersonal zur alltagsintegrierten Sprachbildung und Sprachförderung (HIT)}

Das HIT basiert auf dem „Heidelberger Elterntraining frühe Sprachförderung “ (HET Late Talkers; Buschmann 2017) und richtet sich an pädagogisches Fachpersonal in Kinderkrippen und Kindergärten sowie an Tagesmütter. Es zielt zum einen auf die Erweiterung der sprachförderlichen Kompetenzen des Fachpersonals, zum anderen auf eine Verbesserung der kindlichen Sprachlernmöglichkeiten in natürlichen Interaktionen im Alltag der Kindertageseinrichtungen ab. Der Fokus liegt auf sprachauffälligen Kindern 
(Buschmann et al. 2010; s. hierzu auch Interaktion mit sprachlich auffälligen Kindern).

\section{Interaktion mit sprachlich auffälligen Kindern}

Girolametto et al. (2000) wiesen darauf hin, dass Eltern und pädagogische Fachkräfte gerade im Umgang mit sprachlich auffälligen Kleinkindern den späten Sprechern/Sprecherinnen (Late Talker) einen Interaktionsstil verwenden, der durch viele Anweisungen und wenig sprachmodellierenden Input geprägt ist. Dadurch werden die Möglichkeiten der Kinder zur Wahrnehmung korrekter Sprachvorbilder sowie zum Erhalt korrektiver Rückmeldungen über ihr eigenes Sprechen eingeschränkt (s. hierzu auch - Kap. 13). Eine ähnlich große Herausforderung stellt der sprachliche Umgang mit Kleinkindern dar, die bisher nur wenig Kontakt mit der deutschen Sprache hatten und deshalb über einen geringen Wortschatz verfügen.

Das HIT ist als mehrtägige Fortbildung mit Supervision konzipiert, die in einer Kleingruppe über einen Zeitraum von 6 bis 8 Monaten durchgeführt wird. Sie besteht aus 5 Basisterminen $\mathrm{zu}$ je 5 Unterrichtseinheiten mit $45 \mathrm{~min}$, die im Abstand von 3 bis 4 Wochen stattfinden. Neben einer Wissensvermittlung $\mathrm{zu}$ den Themen „Frühe Sprachentwicklung und deren Störungen“ sowie „Mehrsprachigkeit“ werden die pädagogischen Fachkräfte systematisch darin trainiert, eine sprachförderliche Grundhaltung einzunehmen, 14 sprachmodellierende Strategien anzuwenden und alltägliche Situationen effektiv für die Sprachförderung zu nutzen; Beispiel: Grundprinzipien sprachförderlicher Kommunikation (Buschmann 2017).

\section{Beispiel: Grundprinzipien}

sprachförderlicher Kommunikation

(Buschmann 2017)

- Auf Augenhöhe mit dem Kind begeben.

- Direkte Zuwendung, Blickkontakt aufnehmen.

- Abwarten, was das Kind sagen möchte.

- Dem Kind aufmerksam und interessiert zuhören.
- Das Kind beim Reden nicht unterbrechen.

- Bestätigende Wiederholungen kindlicher Äußerungen.

- Einfache, kurze Sätze verwenden.

- Langsam, deutlich und mit guter Betonung sprechen.

- Eigene Äußerungen wiederholen oder umformulieren.

- Interessiertes Nachfragen unter Verwendung offener W-Fragen: Wie? Warum? Was?

- Spaß am Sprechen vermitteln.

Zur Sicherung des Langzeiteffekts folgt nach etwa 4 Monaten ein Nachschulungstermin mit ebenfalls 5 Unterrichtseinheiten. Für die Altersgruppe der 3- bis 5-jährigen Kinder existiert eine spezifische Adaptation des Trainingsprogramms, in der mit den pädagogischen Fachkräften z. B. das dialogische Vorlesen zusätzlich detailliert erarbeitet wird. Das intensive Üben sprachförderlicher Verhaltensweisen findet in Form von Rollenspielen statt und erfolgt zwischen den Sitzungen mit den Kindern in der Einrichtung. Begleitend werden Videos von in der Kindertageseinrichtung aufgezeichneten Bilderbuchsituationen supervidiert. Dabei werden stets die wichtigsten Grundprinzipien sprachförderlichen Verhaltens berücksichtigt (Buschmann und Sachse 2018).

Befunde einer Pilotstudie mit Prätest-Posttest-Design zur Wirksamkeit des Trainings in Stuttgart und Heidelberg (Buschmann et al. 2010) lassen auf Veränderungen der sprachförderlichen Grundhaltung bei den Fachkräften schließen, die sich allerdings noch nicht durchgängig in ihrem Verhalten niederschlagen. Die Rate der Kinder, die mit 2,5 Jahren den Sprachrückstand kompensiert hatten und im weiteren Entwicklungsverlauf altersgemäße Sprachfähigkeiten zeigten, konnte im Vergleich $\mathrm{zu}$ einer herkömmlichen Fortbildung um das 2,3-Fache erhöht werden. 
Die Stichprobengröße war mit 28 Kindern jedoch sehr klein.

Simon und Sachse $(2011,2013)$ untersuchten zudem die Effektivität des HIT auf das Interaktionsverhalten der pädagogischen Fachkräfte im Rahmen eines randomisierten Prä-Post-Kontrollgruppendesigns mit Follow up. Beim gemeinsamen Betrachten von Bilderbüchern mit sprachlich schwachen 3- bis 5-jährigen Kindern konnte das sprachliche Interaktionsverhalten der Fachkräfte optimiert werden, was sich wiederum positiv auf die Sprechfreude der Kinder und die Komplexität ihrer Äußerungen auswirkte (s. auch Projekt zur alltagsintegrierten Sprachförderung (Jungmann et al. 2013)).

\section{Projekt zur alltagsintegrierten Sprachförderung (Jungmann et al. 2013)}

In einem Projekt zur alltagsintegrierten Sprachförderung für Kinder im Alter von 3;0 bis 5;11 Jahren in Mecklenburg-Vorpommern (Jungmann et al. 2013) bildete das HIT (Buschmann et al. 2010) das Rahmenkonzept. Dieses wurde um 2 Fortbildungseinheiten zur Förderung der phonologischen Bewusstheit und der Grammatik nach dem Konzept des Deutschen Jugendinstituts (DJI) „Kinder-Sprache stärken“ (Jampert et al. 2009) und um die ,alltagsintegrierte Sprachförderung für Kinder mit Migrationshintergrund" (Adler 2011) ergänzt.

Die Wirksamkeit des Trainings auf das sprachförderliche Handeln der Fachkräfte und auf die sprachlichen Kompetenzen der Kinder wurde im Rahmen eines Prätest-Posttest-Designs überprüft. Dabei wurden die Sprachverständnis-, Sprachproduktionsund Sprachgedächtnisleistungen von 263 Kinder jeweils mit dem „Sprachentwicklungstest für dreibis fünfjährige Kinder" (SETK 3-5; Grimm 2010) erfasst. Das sprachliche Interaktionsverhalten der 27 pädagogischen Fachkräfte wurde vor und nach dem Training in der Bilderbuchsituation und beim Freispiel videografiert. Es wurden jeweils die Redeanteile der pädagogischen Fachkräfte und der Kinder ermittelt und die Interaktionen mit einem kategorialen Codierungssystem ausgewertet, das sich auf die Umsetzung zentraler Fortbildungsinhalte, z. B. den Einsatz von Sprachlehrstrategien und das Stellen von sprachanregenden Fragen, bezieht.

Im Prätest wurden rund $20 \%$ der einsprachig deutschen Kinder und mehr als die Hälfte der zweioder mehrsprachig aufwachsenden Kinder als sprachauffällig identifiziert. Über die Zeit zeigten alle
Kinder eine signifikante Verbesserung ihrer sprachlichen Leistung. Ein substanzieller Anteil der Varianz der sprachlichen Leistungen der Kinder im Posttest ließ sich durch die Veränderungen des sprachlichen Interaktionsverhaltens der Fachkräfte in der Bilderbuchsituation, nicht aber im Freispiel erklären. Die Generalisierung der Fortbildungsinhalte, die vor allem auf das dialogische Bilderbuchlesen als Protosituation der alltagsintegrierten Spracheinführung abstellten, gelang in dem sehr kurzen Zeitraum von 6 Monaten vermutlich noch nicht für das freie Spiel. Möglicherweise sind hier Veränderungen der Fortbildungspraxis angezeigt, die z. B. in einer Ergänzung durch ein gezieltes pädagogisches Coaching im Alltag bestehen könnten, um den pädagogischen Fachkräften den Transfer der Fortbildungsinhalte in den pädagogischen Alltag zu erleichtern (Neuman und Cunningham 2009).

\subsubsection{Professionalisierung pädagogischer Fachkräfte im KOMPASS-Projekt}

Vor dem Hintergrund der Bildungskonzeption für Kinder von 0 bis 10 Jahren des Landes Mecklenburg-Vorpommern, aktuellen Modellvorstellungen zur Professionalisierung pädagogischer Fachkräfte (Anders 2012) und den Befunden zu den Einflussfaktoren und Wirkmechanismen auf den Ebenen der Struktur- und Prozessqualität (Tietze et al. 2005) wurde im Rahmen des KOMPASS-Projektes ein umfassendes Professionalisierungskonzept für pädagogische Fachkräfte entwickelt (Jungmann et al. 2015). Ziel war es, die Qualität der Fachkraft-KindInteraktionen in bildungsrelevanten Alltagssituationen $\mathrm{zu}$ erhöhen und damit die Entwicklungs- und Bildungschancen der Kinder zu verbessern und deren Übergang in die Schule zu optimieren.

Die Fachkräfte nahmen gemeinsam an insgesamt $11 \mathrm{~h}$ Grundlagenfortbildung zu folgenden allgemeinen Themen teil:

- Aufgaben und Rolle der pädagogischen Fachkraft

- Fachkraft-Kind-Beziehung

- Erziehung und Bildung

- Alltagsintegrierte Förderung und Resilienz 
- Beobachtung und Dokumentation

- Erziehungspartnerschaften

- Netzwerkarbeit

Darauf aufbauend erhielten die 16 Fachkräfte im Bildungsbereich Sprache und Literacy eine $33 \mathrm{~h}$ umfassende fachspezifische Fortbildung mit den Themenschwerpunkten:

- Sprachentwicklung und -förderung

- Mehrsprachigkeit und Zweitspracherwerb

- Frühe literale Kompetenzen

Um den Nutzen eines Coachings der Fachkräfte zusätzlich zu den Fortbildungen zu erfassen, erhielt die Hälfte der teilnehmenden pädagogischen Fachkräfte $(\mathrm{n}=8) \quad 5-7$ fachspezifische Individualcoachings von 90 bis 180 min in Alltagssituationen in der Kindertageseinrichtung über einen Zeitraum von 6 bis 9 Monaten. In deren Rahmen wurden die Fachkräfte bei der Planung, Durchführung und Auswertung ihrer sprachförderlichen Interaktionen in verschiedenen Alltagssituationen begleitet, um den Transfer der Fortbildungsinhalte im Hinblick auf eine erfolgreiche Unterstützung der Entwicklungs- und Lernprozesse der Kinder in die Praxis zu optimieren (Oelkers und Reusser 2008). Um Möglichkeiten der alltagsintegrierten Förderung aller Kinder für die pädagogischen Fachkräfte sichtbarer zu machen, wurden in den Fortbildungen und Individualcoachings die Situationswahrnehmung und -analyse anhand von Fall- und Situationsbeispielen aus der Begrüßungs-, der Mittags- und der Bilderbuchsituation thematisiert.

Zur Überprüfung der Effektivität des Professionalisierungskonzepts wurde die Umsetzungsqualität der Fortbildungen und Coachings mit Dokumentations-, Fragebögen und verschiedenen Interviewformen erfasst. Die Effekte auf das sprachspezifische Wissen wurden mit dem „Fragebogen zur
Erfassung von sachbezogenen Kompetenzen von Fachkräften in der Frühpädagogik“ (FESKO-F; Hendler et al. 2011) zu 3 Messzeitpunkten erfasst, ebenso wie die Einstellungen der Fachkräfte und deren Handlungskompetenzen in standardisierten oder halbstandardisierten Alltagssituationen in der Kindertageseinrichtung (Bilderbuchsituation, Begrüßungssituation, gemeinsames Mittagessen). Diese wurden videografiert und sowohl qualitativ als auch quantitativ ausgewertet.

Die Effekte der Professionalisierungsmaßnahmen auf die kindliche Entwicklung wurden ebenfalls zu 3 Messzeitpunkten mit standardisierten Tests - im Bildungsbereich Sprache mit dem SETK 3-5 (Grimm 2010) und im Bildungsbereich Literacy mit dem Test „Erzähl-und Lesekompetenzen erfassen bei 3- bis 5-jährigen Kindern" (EuLe 3-5; Meindl und Jungmann 2014) direkt bei den Kindern erhoben.

Um der Komplexität des Bedingungsgefüges gerecht $\mathrm{zu}$ werden, wurden Daten zu zahlreichen Einflussfaktoren auf der Ebene der Kindertageseinrichtung (z. B. Struktur- und Prozessqualität), bei den Fachkräften (z. B. Persönlichkeit, pädagogische Orientierung, Arbeitsbelastung) und den Kindern (nonverbale Intelligenz, sozioökonomischer Status) gesammelt, um diese bei der Beantwortung der Frage, ob die Professionalisierungsmaßnahmen zu einer verbesserten kindlichen Entwicklung und Bildung führen, mit berücksichtigen zu können.

Die begleitende Wirksamkeitsstudie zum KOMPASS-Projekt ergab, dass das Fachwissen der Fachkräfte über den Bildungsbereich Sprache durch die Fortbildungen signifikant gesteigert werden konnte (Jungmann und Koch 2017). Weiterhin war eine Zunahme sprachförderlicher Verhaltensweisen in der Bilderbuchsituation und beim gemeinsamen 
Mittagessen zu konstatieren. Die T-Werte der an dieser Studie beteiligten 326 Kinder im SETK 3-5 nahmen über die Zeit signifikant im Mittel um etwa eine halbe Standardabweichung von 45 auf 50 T-Wert-Punkte zu. Der recht hohe Anteil von rund $25 \%$ Risikokindern $(\mathrm{n}=82)$ stammte überwiegend aus Familien mit schwachem sozioökonomischem Status $(22,6 \%)$, wohingegen lediglich $12,7 \%$ der sprachauffälligen Kinder aus Elternhäusern mit mittlerem oder hohem Sozialstatus kamen. Nach den fachspezifischen Fortbildungen der Fachkräfte war ein Rückgang auf $18 \%$ Risikokinder $(n=57)$ $\mathrm{zu}$ verzeichnen. Insbesondere der Anteil der sprachauffälligen Kinder aus Familien mit einem mittleren sozioökonomischen Status ging deutlich auf 10,5\% zurück. Neben der Kindertagesstättenqualität, dem Fachwissen der pädagogischen Fachkräfte im Bereich Sprache und ihrer Teilnahme an der Fortbildung und dem Coaching waren auch die Persönlichkeit und das Belastungserleben der Fachkräfte wichtige Prädiktoren der sprachlichen Kompetenzen der Kinder, die stärkere Beachtung in der Fortbildungskonzeption erfahren sollten (Jungmann und Koch 2017).

\subsection{Implikationen für die Praxis}

Die zentrale Bedeutung einer elementaren, alltagsintegrierten Sprachförderung hat inzwischen Eingang in die Bildungskonzeptionen aller Bundesländer gefunden ( Abschn. 14.2). Diese Form der Sprachförderung stellt allerdings hohe Anforderungen an die Fachkräfte, die ausreichendes Wissen über den normalen Spracherwerb und die zugrunde liegenden Erwerbsprozesse benötigen, um Auffälligkeiten wahrzunehmen (Beobachtung und Dokumentation), um sprachförderliche Situationen im Alltag identifizieren zu können und um selbst als Sprachmodell zu fungieren und somit diese Situationen auch effektiv zur adaptiven Sprachförderung zu nutzen (Beckerle und Mackowiak 2019).

In der Praxis kommt es also letztlich darauf an, wie Wissen und Konzepte zur Sprachförderung von den pädagogischen Fachkräften umgesetzt werden, d. h., wie diese mit den Kindern sprechen, wie sie Dialoge führen, wie sie Gespräche mit Kindern gestalten und Instrumente und Material einsetzen, worauf sie ihre Aufmerksamkeit lenken und worauf sie Wert legen. Dies alles hängt von der eigenen sprachlichen Kompetenz und der Sprechfreude der Fachkräfte, ihrer Bildungsbiografie und beruflichen Sozialisation und resultierend daraus ihrer jeweiligen spezifischen Orientierung und Haltung gegenüber dem Bildungsgut Sprache und Schrift ab. Situationen und Handlungsanforderungen in Kindertageseinrichtungen sind hoch komplex, mehrdeutig und nicht standardisierbar.

Kreatives und selbstreflexives Handeln in diesen Situationen wird als professionelle Kompetenz bezeichnet. Wesentlich für den Erfolg von Sprachförderung - dies ist aus den obigen Ausführungen und dem Stand der Forschung deutlich geworden - ist die Sprachförderkompetenz der Fachkräfte. Sprachförderkompetenz bedeutet „ein Zusammenwirken von Wissen, Motiven und Können, das sich im erfolgreichen pädagogischen Handeln in spezifischen beruflichen Bereichen zeigt und weiterentwickelt" (Fried und Briedigkeit 2008, S. 10).

Um diese Aufgaben umfassend bewältigen $\mathrm{zu}$ können, müssen die pädagogischen Fachkräfte einerseits in ihren diagnostischen Kompetenzen geschult, andererseits aber auch befähigt werden, als sprachliches Vorbild $\mathrm{zu}$ fungieren und sich und ihr kommunikationsförderndes Verhalten stets zu reflektieren (Jungmann und Albers 2013; Jungmann et al. 2015). 
Wenngleich die Bildungspläne keine spezifischen Vorgaben enthalten, wie die Sprachförderkompetenz der Fachkräfte zu entwickeln ist, liegen mittlerweile 2 Instrumente zur Selbst- und Teamqualifizierung vor: die ,Dortmunder Ratingskala zur Erfassung sprachförderrelevanter Interaktionen" (DO-RESI; Fried und Briedigkeit 2008) und das Beobachtungsverfahren „Literacy und Sprache in Kindertageseinrichtungen" (LiSKit; Mayr et al. 2012).

\subsubsection{Erfassung sprachförderrelevanter Interaktionen}

\subsubsection{Dortmunder Ratingskala zur Erfassung sprachförderrelevanter Interaktionen (DO-RESI)}

Die DO-RESI (Fried und Briedigkeit 2008) ist ein wissenschaftlich fundiertes Verfahren zur Erfassung sprachförderrelevanter Interaktionen in der Kindertageseinrichtung, das als Selbst- und Teamqualifizierungsinstrument dienen soll. Es werden sprachförderrelevante Prozesse auf 4 Ebenen oder Qualitätsdimensionen (Organisation, Beziehung, adaptive

14 Unterstützung und sprachlich-kognitive Herausforderung) mit insgesamt 23 Items (Einschätzaufgaben) erfasst, die • Tab. 14.1 im Überblick zeigt.

Das Verhalten der pädagogischen Fachkraft wird nach der Hospitation für jedes Item in 7 Qualitätsstufen von „unzureichend“ bis ,exzellent“ eingeschätzt und in einem Protokollbogen angekreuzt. Um die Einschätzung zu objektivieren, gibt es Indikatoren, die Interaktionsmerkmale beschreiben, die für die jeweilige Qualitätsstufe charakteristisch sind.

Unabdingbar für die objektive Durchführung dieses Verfahrens sind ein vorgeschaltetes Training und die Erprobung in der Praxis. Dafür liegt dem Instrument eine DVD mit Beobachtungsaufgaben bei. Allerdings scheint im Alltag eine Beobachtung derselben Fachkraft durch 2 Beobachtende über einen Zeitraum von $3 \mathrm{~h}$ in der Erprobungsphase kaum realisierbar. Die retrospektive Einschätzung aller Items ist schwierig, da während der Hospitation lediglich Notizen erlaubt sind. Schließlich bleibt anzumerken, dass die Frage nach den Konsequenzen, um anschließend positiv auf die Interaktionsqualität Einfluss zu nehmen, unbeantwortet bleibt.

\subsubsection{Beobachtungsverfahren Literacy und Sprache in Kindertageseinrich- tungen (LiSKit)}

Das Beobachtungsverfahren LiSKit (Mayr et al. 2012) ist ebenfalls ein Instrument für pädagogische Fachkräfte, um die Qualität sprachlicher Bildung in Kindertageseinrichtungen $\mathrm{zu}$ evaluieren und $\mathrm{zu}$ optimieren. Schwerpunkt der Beobachtung sind pädagogische Fachkräfte bei der Arbeit mit Kindern im Alter von 3 bis 6 Jahren.

Das 56-seitige Begleitheft zu den Bögen enthält Hinweise zu Aufbau, Konzeption und Nutzung des Bogens, Grundlagenwissen zur sprachlichen Bildung in Kindertageseinrichtungen, theoretische Hintergründe zu den einzelnen Themenbereichen und Bausteinen sowie Arbeitsschemata zur Qualitätsentwicklung im Hinblick auf Sprache und Literacy. Der 32-seitige Bogen umfasst 6 Bereiche, die wiederum in insgesamt 26 Bausteine unterteilt sind, wie $\bullet$ Tab. 14.2 zeigt.

Das Verfahren kann zur internen und externen Evaluation genutzt und von einzelnen oder mehreren Fachkräften gemeinsam bearbeitet werden. Dies liefert ein umfangreiches Bild über die Qualität der Kindertageseinrichtung für den Bildungsbereich Sprache sowie erstmals auch für den Bildungsbereich Literacy. 
Tab. 14.1 Die 4 Qualitätsdimensionen von DO-RESI mit den zugehörigen Items (Fried und Briedigkeit 2008, S. 17)

\begin{tabular}{|c|c|}
\hline Qualitätsdimension & Items \\
\hline Organisation & $\begin{array}{l}\text { - Pädagogischer Überblick } \\
\text { - Planung, Routine } \\
\text { - Vorkehrungen für Kinder mit besonderem Sprachförder- } \\
\text { bedarf } \\
\text { - Sprachfördergewohnheiten } \\
\text { - Gesprächsförderung }\end{array}$ \\
\hline Beziehung & $\begin{array}{l}\text { - Kontrolle } \\
\text { - Kongruenz } \\
\text { - Empathisches Zuhören } \\
\text { - Nähe }\end{array}$ \\
\hline Adaptive Unterstützung & $\begin{array}{l}\text { - Sensitivität } \\
\text { - Engagement } \\
\text { - Anregung } \\
\text { - Handlungen verbalisieren } \\
\text { - Verständnissicherung } \\
\text { - Informationen, Berichte einholen } \\
\text { - Instruktionswechsel } \\
\text { - Lernmöglichkeiten aufzeigen }\end{array}$ \\
\hline Sprachlich-kognitive Herausforderung & $\begin{array}{l}\text { - Vielfalt des Wortschatzes } \\
\text { - Grammatikalisch komplexer Input } \\
\text { - Offene Fragen } \\
\text { - Themen benennen, entdecken } \\
\text { - Themen verbinden } \\
\text { - Zusammenhänge eines Themas klären, hinterfragen }\end{array}$ \\
\hline
\end{tabular}

Positiv sind die weiterführenden Anregungen für die Umsetzung von Sprach- und Literacy-Förderung in die Praxis zu bewerten. Da die Bögen variabel nach den individuellen zeitlichen Möglichkeiten und Interessen der Fachkräfte ausgefüllt werden können, ist die Bearbeitung gut in den pädagogischen Alltag integrierbar (Jungmann und Albers 2013).

\subsubsection{Erforderliche Kompetenzen aufseiten der Fachkräfte}

Zusammengenommen ist $\mathrm{zu}$ konstatieren, dass die Anforderungen an pädagogische Fachkräfte hoch sind, wenn alltagsintegrierte Sprachförderung gelingen soll (Hopp et al. 2011; List 2010).
Um Auffälligkeiten wahrnehmen, sprachförderliche Situationen im Alltag identifizieren, selbst als Sprachvorbild fungieren und somit Situationen auch effektiv zur adaptiven Sprachförderung nutzen zu können, benötigen pädagogische Fachkräfte umfassende diagnostische Kenntnisse, um geeignete Instrumente auszuwählen und daraus Schlussfolgerungen für die Auswahl, Planung und Erfolgskontrolle von Sprachförderung zu ziehen. Sie sollten bei der Durchführung, der Auswertung und der Interpretation der Ergebnisse sicher sein sowie standardisierte Anweisungen bei den Verfahren beachten (vgl. - Kap. 7 sowie $>$ Kap. 8).

Nur vor dem Hintergrund von entwicklungspsychologischem Wissen zum normalen Spracherwerb und den zugrunde liegenden Erwerbsprozessen vermögen 
- Tab. 14.2 Beobachtungsbereiche und Bausteine von LiSKit (Mayr et al. 2012)

\begin{tabular}{|c|c|}
\hline Beobachtungsbereich & Bausteine \\
\hline Ausstattung und Räumlichkeiten & $\begin{array}{l}\text { - Buchbereich/Raum für Bücher } \\
\text { - Buchbestand } \\
\text { - Medien und Technik für Kinder } \\
\text { - Schreiben und Schriftkultur } \\
\text { - Gesellschaftsspiele } \\
\text { - Rollenspiele }\end{array}$ \\
\hline Aktivitäten der Fachkraft & $\begin{array}{l}\text { - Gespräche mit Kindern } \\
\text { - Anregung von Gesprächen unter Kindern } \\
\text { - Lieder/Reime/Spiele } \\
\text { - Bilderbuchbetrachtung/Vorlesen/Hörbücher } \\
\text { - Rund ums Buch } \\
\text { - Schriftkultur/,Schreiben“ } \\
\text { - „Lesen“ im Rollenspiel }\end{array}$ \\
\hline $\begin{array}{l}\text { Interaktion in ausgewählten Situationen: Selbst- } \\
\text { einschätzung }\end{array}$ & $\begin{array}{l}\text { - Bilderbuchbetrachtungen } \\
\text { - Vorlesen } \\
\text { - Freie Gespräche in Gesprächsrunden, Gespräche } \\
\text { bei naturwissenschaftlichen Themen/Projekten }\end{array}$ \\
\hline $\begin{array}{l}\text { Interaktion in ausgewählten Situationen: kollegiale } \\
\text { Beobachtung }\end{array}$ & $\begin{array}{l}\text { - Bilderbuchbetrachtungen } \\
\text { - Vorlesen } \\
\text { - Freie Gespräche in Gesprächsrunden, Gespräche } \\
\text { bei naturwissenschaftlichen Themen/Projekten }\end{array}$ \\
\hline Spracherfassung, Konzeption und Qualifizierung & $\begin{array}{l}\text { - Beobachtung und Dokumentation von Sprache } \\
\text { und Literacy } \\
\text { - Verankerung von Sprache und Literacy in der } \\
\text { Einrichtung } \\
\text { - Qualifizierung des pädagogischen Personals }\end{array}$ \\
\hline Zusammenarbeit und Vernetzung & $\begin{array}{l}\text { - Bildungs- und Erziehungspartnerschaft mit Eltern } \\
\text { - Vernetzung beim Thema sprachliche Bildung } \\
\text { - Vernetzung bei Kindern mit Problemen im sprach- } \\
\text { lichen Bereich }\end{array}$ \\
\hline
\end{tabular}

sie, altersgemäße Erwerbsprozesse in der Erst- und der Zweitsprache einzuschätzen, Phänomene der Kindersprache den einzelnen Ebenen zuzuordnen und entsprechend Schwerpunkte der alltagsintegrierten Förderung darauf abzustimmen (vgl. Kap. 1 sowie - Kap. 5). Für den Erwerb von Dialog- und Diskursfähigkeiten ist die Eingewöhnung in Standardmuster interaktiver Handlungen erforderlich: Sprecherwechsel bei Fragen, Auffordern, Erklären, Rechtfertigen, Widerspruch einlegen. Darüber hinaus benötigen sie Wissen über Zusammenhänge des Spracherwerbs mit anderen Bereichen der kindlichen Entwicklung. Insbesondere die wechselseitige Beeinflussung kognitiver, sozialer und sprachlicher Kompetenzen sollte bekannt sein (vgl. > Kap. 6). Wenn sich pädagogische Fachkräfte allein auf die Sprachproduktion der Kinder konzentrieren, droht die Unterstützung des Spracherwerbs zum Training zu werden.

Weiterhin sollten pädagogische Fachkräfte einen Überblick über die wichtigen existierenden Förderkonzepte haben ( Abschn. 14.3) sowie über Kriterien zu ihrer Beurteilung verfügen. Eine 
Orientierung geben die Befunde aus vorliegenden narrativen Reviews (Egert und Hopf 2016) und der Qualitätscheck der BiSS-Initiative.

Werden Förderprogramme durchgeführt, ist auf entsprechende Programmtreue und die begleitende Dokumentation zu achten, um eine hohe Umsetzungsqualität sicherzustellen. Dabei sollten die Förderbedürfnisse einzelner Kinder Berücksichtigung finden. Die Kinder sollten zum Erzählen angeregt werden, indem die Fachkräfte passende Materialien bereitstellen, sprachanregende Rückfragen stellen und angemessene Rückmeldungen geben. Dazu gehört auch, sie zur Beachtung der Besonderheiten verschiedener Texttypen (Tatsachenbericht, fiktive Erzählung, Erzählung eigener Erlebnisse) aufzufordern. Das gemeinsame Betrachten von Bilderbüchern und der dialogische Austausch über deren Inhalte bilden ebenso wie der tägliche Umgang mit Schrift (z. B. in Büchern, Zeitungen, Schriftzügen in der Werbung, dem eigenen Namen) die Motivationsgrundlage für den späteren Schriftspracherwerb.

Kindertageseinrichtungen kommt häufig kompensatorisches Potenzial zu, wenn frühe Erfahrungen mit Schrift zu Hause nicht in ausreichendem Maße gemacht werden können. Damit eröffnet sich gleichzeitig ein wichtiges Feld der Elternpartizipation (vgl. $>$ Kap. 13).

Um dieser Vielfalt an Anforderungen gerecht werden zu können, sollten pädagogische Fachkräfte bereit sein, die Wirkungen ihrer Arbeit begutachten zu lassen und sich selbstkritisch an internen Evaluationen $\mathrm{zu}$ beteiligen. Die Unterstützung bei der praktischen Umsetzung von additiver oder alltagsintegrierter Sprachförderung durch ein pädagogisches Coaching oder kollegiale Intervision kann nur dann die gewünschte Wirkung entfalten, wenn das ganze Team zur Kooperation bereit ist und
Verbindlichkeit herrscht. Somit erweist sich Sprachförderung in Kindertageseinrichtungen auch als eine umfassende Managementaufgabe, die von den Leitungspersonen und den Trägern wahrgenommen werden muss (Jungmann und Albers 2013; Strehmel 2010).

\subsection{Zusammenfassung}

- Entlang der Dimension Orientierungsqualität wurden Gemeinsamkeiten und Unterschiede der Bildungspläne der Länder zur Sprachbildung und -förderung skizziert.

- Spezifischere Anhaltspunkte zur konkreten Umsetzung der Sprachförderung in Kindertageseinrichtungen und damit zur Erhöhung der Prozessqualität enthalten Sprachförderkonzepte, wobei zwischen sprachstrukturellen (additiven) und ganzheitlichen (alltagsintegrierten) Sprachförderkonzepten unterschieden wird.

- Exemplarisch wurden Konzepte vorgestellt und Befunde zu deren Effektivität berichtet. Herausforderungen an die Sprachförderkompetenzen der Fachkräfte werden abgeleitet und Möglichkeiten der Selbst- und Teamreflexion aufgezeigt.

- Es bleibt zu konstatieren, dass dem vergleichsweise breiten Wissen darüber, wie Kinder in ihrer sprachlichen Kompetenz gefördert werden können, ein noch großer Forschungs- und Entwicklungsbedarf bei der Umsetzung dieses Wissens durch die pädagogischen Fachkräfte in die Praxis gegenübersteht.

\subsection{Weiterführende Literatur}

Mehr zum allgemeinen Hintergrund und zur Rahmung des Beitrags kann nachgelesen werden in: 
- Jungmann, T., \& Albers, T. (2013). Frühe sprachliche Bildung und Förderung. München: UTB \& Reinhardt.

Praktische Anregungen und eine Sammlung von 60 Spielen zur alltagsintegrierten Sprach- und Literacy-Förderung im Kindergarten finden sich bei:

- Jungmann, T., Morawiak, U., \& Meindl, M. (2016). Überall steckt Sprache drin. Alltagsintegrierte Sprach- und Literacyförderung für 3- bis 6-jährige Kinder. München: Reinhardt.

Eine ausführliche Darstellung des KOMPASS-Projektes und seiner Evaluationsergebnisse bietet der Herausgeberband von Jungmann und Koch (2017):

- Jungmann, T., \& Koch, K. (Hrsg.). (2017). Professionalisierung pädagogischer Fachkräfte in Kindertageseinrichtungen. Wiesbaden: Springer.

Eine Übersicht über die Wirkungen vorschulischer Sprachförderung mit einem besonderen Fokus auf praktische Implikationen gibt das Handbuchkapitel von Egert (2016):

- Egert, F. (2016). Wirkung vorschulischer Sprachförderung - Stolpersteine und Praxisimplikationen. In R. Sigel \&

E. Inckemann (Hrsg.), Diagnose und Förderung von Kindern mit Zuwanderungshintergrund in Sprach- und Schriftsprachenerwerb (S. 65-78). Bad Heilbrunn: Klinkhardt.

\section{Literatur}

Adler, Y. (2011). Kinder lernen Sprache(n): Alltagsorientierte Sprachförderung in der Kindertagesstätte. Stuttgart: Kohlhammer.

Anders, Y. (2012). Modelle professioneller Kompetenzen für frühpädagogische Fachkräfte. Expertise zum Gutachten „Professionalisierung in der Frühpädagogik“. \ http://www.aktionsrat-bildung.de/fileadmin/Dokumente/Expertise_ Modelle_professioneller_Kompetenzen.pdf. Zugegriffen: 20. Juli 2015.
Bayerisches Staatsministerium für Arbeit und Sozialordnung, Familie und Frauen. (2016). Der Bayerische Bildungs- und Erziehungsplan für Kinder in Tageseinrichtungen bis zur Einschulung (7. Aufl.). Berlin: Cornelsen.

Beckerle, C., \& Mackowiak, K. (2019). Adaptivität von Sprachförderung im Kita-Alltag. Ein Vergleich des Sprachförderhandelns pädagogischer Fachkräfte bei Kindern mit Deutsch als Erstund Zweitsprache und unterschiedlichen Sprachkompetenzen. Lernen und Lernstörungen, 8, 1-9.

- https://doi.org/10.1024/2235-0977/a000270.

Behörde für Arbeit, Soziales, Familie und Integration, Hamburg. (2012). Hamburger Bildungsempfehlungen für die Bildung und Erziehung von Kindern in Kindertageseinrichtungen. 2. Überarbeitete Auflage. http://www.hamburg. de/kita/116828/bildungsempfehlungen.html. Zugegriffen: 6. Mai 2018.

BiSS-Trägerkonsortium. (2020). „Bildung durch Sprache und Schrift" (BiSS). $>$ https://bisssprachbildung.de/ueber-biss/. Zugegriffen: 6 . März 2020.

Brunner, M., \& Schöler, H. (2002). Heidelberger Auditives Screening in der Einschulungsuntersuchung HASE (1. Aufl.). Göttingen: Hogrefe.

Buschmann, A. (2017). Heidelberger Elterntraining frühe Sprachförderung. HET Late Talkers (3. Aufl.). München: Elsevier.

Buschmann, A., \& Sachse, S. (2018). Heidelberg interaction training for language promotion in early childhood settings (HIT). European Journal of Education, 53(1), 66-78.

Buschmann, A., Simon, S., Jooss, B., \& Sachse, S. (2010). Ein sprachbasiertes Interaktionstraining für ErzieherInnen („Heidelberger Trainingsprogramm") zur alltagsintegrierten Sprachförderung in Krippe und Kindergarten - Konzept und Evaluation. In K. Fröhlich-Gildhoff, I. Nentwig-Gesemann, \& P. Strehmel (Hrsg.), Forschung in der Frühpädagogik III, Schwerpunkt: Sprachentwicklung \& Sprachförderung. Materialien zur Frühpädagogik (Bd. 5, S. 107-133). Freiburg: FEL.

Egert, F. (2016). Wirkung vorschulischer Sprachförderung - Stolpersteine und Praxisimplikationen. In R. Sigel \& E. Inckemann (Hrsg.), Diagnose und Förderung von Kindern mit Zuwanderungshintergrund in Sprach- und Schriftsprachenerwerb (S. 65-78). Bad Heilbrunn: Klinkhardt.

Egert, F., \& Hopf, M. (2016). Zur Wirksamkeit von Sprachförderung in Kindertageseinrichtungen in Deutschland. Ein narratives Review. Kindheit und Entwicklung, 25, 153-163.

European Child Care and Education Study Group (ECCE). (1999). European Child care and education study: School-age assessment of child 
development: Long-term impact of pre-school experiences in school success, and family-school relationships. FU Berlin: ECCE.

Frank, G., \& Grziwotz, P. (2001). Lautprüfbogen. Ravensburg: Sprachheilzentrum.

Fricke, S., \& Schäfer, B. (2011). Test für Phonologische Bewusstheit TPB (2. Aufl.). Idstein: SchulzKirchner.

Fried, L., \& Briedigkeit, E. (2008). Sprachförderkompetenz - Selbst- und Teamqualifizierung für Erzieherinnen, Fachberatungen und Ausbilder. Berlin: Cornelsen Scriptor.

Fröhlich, L. P., Metz, D., \& Petermann, F. (2008). Kindergartenbasierte Förderung der phonologischen Bewusstheit „Lobo vom Globo“. Kindheit und Entwicklung, 18(4), 204-212.

Fröhlich, L. P., Metz, D., \& Petermann, F. (2010). Förderung der phonologischen Bewusstheit und sprachlicher Kompetenzen: Das LoboKindergartenprogramm. Göttingen: Hogrefe.

Girolametto, L., Weitzmann, E., van Lieshout, R., \& Duff, D. (2000). Directiveness in teachers' language input to toddlers and preschoolers in day care. Journal of Speech, Language, and Hearing Research, 43(5), 1101-1114.

Grimm, H. (2010). Sprachentwicklungstest für drei- bis fünfjährige Kinder SETK 3-5 (2. Aufl.). Göttingen: Hogrefe.

Grimm, H., \& Schöler, H. (1991). Heidelberger Sprachentwicklungstest HSET (2. Aufl.). Göttingen: Hogrefe.

Hendler, J., Mischo, C., Strohmer, J., \& Wahl, S. (2011). Das sprachbezogene Wissen angehender pädagogischer Fachkräfte im Wissenstest und in der Selbsteinschätzung. Empirische Pädagogik, 25(4), 518-542.

Hopp, H., Thoma, D., \& Tracy, R. (2011). Sprachförderkompetenz pädagogischer Fachkräfte. Ein sprachwissenschaftliches Modell. Zeitschrift für Erziehungswissenschaft, 13(4), 609-629.

Jampert, K., Best, P., Guadatiello, A., Holler, D., \& Zehnbauer, A. (2007). Schlüsselkompetenz Sprache. Sprachliche Bildung und Förderung im Kindergarten. Konzepte, Projekte und Maßnahmen. Weimar: Das Netz.

Jampert, K., Zehnbauer, A., Best, P., Sens, L. K., \& Laier, M. (2009). Kinder-Sprache stärken! Sprachliche Förderung in der Kita. Wismar: Das Netz.

Jansen, H., Mannhaupt, G., Marx, H., \& Skowronek, H. (2002). Bielefelder Screening zur Früherkennung von Lese-Rechtschreibschwierigkeiten BISC (2. Aufl.). Göttingen: Hogrefe.

Jungmann, T. (2009). Sprachförderung. In A. Lohaus \& H. Domsch (Hrsg.), Psychologische Förderund Interventionsprogramme für das Kindes- und Jugendalter (S. 63-74). Berlin: Springer.
Jungmann, T., \& Albers, T. (2013). Frühe sprachliche Bildung und Förderung. München: UTB \& Reinhardt.

Jungmann, T., \& Koch, K. (Hrsg.). (2017). Professionalisierung pädagogischer Fachkräfte in Kindertageseinrichtungen - Konzept und Wirksamkeit des KOMPASS-Projektes. München: Springer.

Jungmann, T., Koch, K., \& Etzien, M. (2013). Effektivität alltagsintegrierter Sprachförderung bei ein- und zwei- bzw. mehrsprachig aufwachsenden Vorschulkindern. Frühe Bildung, 2(3), 110-121.

Jungmann, T., Morawiak, U., \& Meindl, M. (2015). Überall steckt Sprache drin. Alltagsintegrierte Sprach- und Literacyförderung für 3- bis 6-jährige Kinder. München: Reinhardt.

Kaltenbacher, E., \& Klages, H. (2005). Sprachförderung im Vorschulalter. Entwicklung und Erprobung eines Programms zur sprachlichen Integration von Vorschulkindern. Heidelberg: Institut für Deutsch als Fremdsprachenphilologie.

Küspert, P., \& Schneider, W. (2008). Hören, lauschen, lernen. Sprachspiele für Kinder im Vorschulalter. Das Würzburger Trainingsprogramm zur Vorbereitung auf den Erwerb der Schriftsprache. Göttingen: Vandenhoeck \& Ruprecht.

Lisker, A. (2011). Additive Maßnahmen zur vorschulischen Sprachförderung in den Bundesländern. Expertise im Auftrag des Deutschen Jugendinstituts. \ https://www.dji.de/fileadmin/ user_upload/bibs/Expertise_Sprachfoerderung Lisker_2011.pdf. Zugegriffen: 24. Juli 2015.

List, G. (2010). Frühpädagogik als Sprachförderung. Qualitätsanforderungen für die Aus- und Weiterbildung der Fachkräfte. WiFF-Expertise Nr. 2. - http://www.weiterbildungsinitiative.de/uploads/ media/List.pdf. Zugegriffen: 27. Juli 2015.

Mayr, T., \& Ulich, M. (2010). Der Beobachtungsbogen "Seldak" - Theoretische und empirische Grundlagen. In K. Fröhlich-Gildhoff, I. NentwigGesemann, \& P. Strehmel (Hrsg.), Forschung in der Frühpädagogik III, Schwerpunkt: Sprachentwicklung \& Sprachförderung. Materialien zur Frühpädagogik (Bd. 5, S. 75-106). Freiburg: FEL.

Mayr, T., Hofbauer, C., Kofler, A., \& Šimić, M. (2012). Literacy und Sprache in Kindertageseinrichtungen LisKit. Freiburg: Herder.

Meindl, M., \& Jungmann, T. (2014). Erfassung der frühen Erzähl- und Lesekompetenzen im Vorschulalter zur primären Prävention von Schwierigkeiten im Schriftspracherwerb. Empirische Sonderpädagogik, 3, 211-226.

Ministerium für Arbeit und Soziales des Landes Sachsen-Anhalt. (2014). Bildungsprogramm für Kindertageseinrichtungen in Sachsen-Anhalt. Bildung: Elementar - Bildung von Anfang an. Weimar: Das netz. 
Ministerium für Bildung, Jugend und Sport, Land Brandenburg. (2006). Gemeinsame Erklärung zu Grundsätzen elementarer Bildung in Einrichtungen der Kindertagesbetreuung im Land Brandenburg. $\longrightarrow$ https://mbjs.brandenburg.de/ media/lbm1.c.312232.de. Zugegriffen: 6. Mai 2018.

Ministerium für Bildung, Jugend und Sport, Thüringen. (2015). Thüringer Bildungsplan bis 18 Jahre. Bildungsansprüche von Kindern und Jugendlichen. - http://www.thueringen.de/mam/ th2/tmbwk/bildung/bildungsplan/thuringer_ bildungsplan-18_web.pdf. Zugegriffen: 6. Mai 2018.

Ministerium für Bildung, Wissenschaft und Kultur Mecklenburg-Vorpommern. (2011). Bildungskonzeption für 0 - bis 10-jährige Kinder in Mecklenburg-Vorpommern. Zur Arbeit in Kindertageseinrichtungen und Kindertagespflege. \ttps://www.bildung-mv.de/export/sites/ bildungsserver/downloads/Bildungskonzeptionfuer-0-bis-10-jaehrige-Kinder-in-MecklenburgVorpommern.pdf. Zugegriffen: 6. Mai 2018.

Ministerium für Soziales, Gesundheit, Familie und Gleichstellung des Landes Schleswig-Holstein. (2012). Erfolgreich starten Leitlinien zum Bildungsauftrag von Kindertageseinrichtungen. 5. Aufl. \http://www.schleswig-holstein.de/ DE/Fachinhalte/K/kindertageseinrichtungen/ downloads/kindertageseinrichtungen_ Bildungsauftrag_LeitlinienBildungsauftrag_ B i $1 \mathrm{~d} \mathrm{u} \mathrm{n} \mathrm{g} \mathrm{s} \mathrm{a} \mathrm{u}$ f $\mathrm{r}$ a g L e i t 1 i n i e n . pdf?_blob=publicationFile\&v $=1$. Zugegriffen: 6 . Mai 2018.

Neuman, S. B., \& Cunningham, L. (2009). The impact of professional development and coaching on early language and literacy instructional practices. American Educational Research Journal, 46(2), 532-566.

14 Niedersächsisches Kultusministerium. (2005). Orientierungsplan für Bildung und Erziehung im Elementarbereich niedersächsischer Tageseinrichtung für Kinder. $\triangleright$ https://www. mk.niedersachsen.de/startseite/fruehkindliche_ bildung/orientierungsplan/orientierungsplan-fuerbildung-und-erziehung-86998.html. Zugegriffen: 6. Mai 2018.

OECD. (2001). Starting strong: Early childhood education and care. www.oecd-library.org. Zugegriffen: 20. Juli 2015.

OECD. (2006). Starting strong II: Early childhood education and care. www.oecd-library.org. Zugegriffen: 20. Juli 2015.

OECD. (2011). Starting strong III: A quality toolbox for early childhood education and care. $\$$ www. oecd-library.org. Zugegriffen: 20. Juli 2015.

Oelkers, J., \& Reusser, K. (2008). Qualität entwickeln - Standards sichern - Mit Differenzen umgehen.
Bundesministerium für Bildung und Forschung. Bildungsforschung Band 27. http://www.bmbf. de/pub/bildungsforschung_band_siebenundzwanzig.pdf. Zugegriffen: 19. Juli 2015.

Penner, Z. (2003). Forschung für die Praxis. Neue Wege der sprachlichen Förderung von Migrantenkindern. CH-Berg: Kon-lab GmbH.

Penner, Z., \& Krügel, C. (2007). Programmhandbuch zur „Sprache und frühkindliche Bildung“. Troisdorf: Bildungsverlag Eins.

Petermann, F. (2015). Alltagsintegrierte Förderung oder Förderprogramme im Vorschulalter? Frühe Bildung, 4(3), 161-164.

Raven, J. C. (2002). Coloured progressive matrices $(C P M)$. Bern: Huber.

Reich, H., \& Roth, H.-J. (2004). Hamburger Verfahren zur Analyse des Sprachstandes bei 5-Jährigen (HAVAS-5). Auswertungsbogen Katze und Vogel - Deutsch. Hamburg: Behörde für Bildung und Sport.

Schmid-Barkow, I. (1999). „Phonologische Bewusstheit" als Teil der metasprachlichen Entwicklung im Kontext von Spracherwerbsprozessen und Spracherwerbsstörungen. Die Sprachheilarbeit, 44, 307-317.

Schneider, W. (2018). Nützen Sprachförderprogramme im Kindergarten, und wenn ja, unter welchen Bedingungen? Zeitschrift für Pädagogische Psychologie, 32(1-2), 53-74.

Schneider, W., Visé, M., Reimers, P., \& Blaesser, B. (1994). Auswirkungen eines Trainings der sprachlichen Bewusstheit auf den Schriftspracherwerb in der Schule. Zeitschrift für Pädagogische Psychologie, 8, 177-188.

Schneider, W., Küspert, P., Roth, E., Visé, M., \& Marx, H. (1997). Short- and long-term effects of training phonological awareness in kindergarten: Evidence from two German studies. Journal of Experimental Child Psychology, 66, 311-340.

Schneider, W., Roth, E., \& Ennemoser, M. (2000). Training phonological skills and letter knowledge in children at risk for dyslexia: A comparison of three kindergarten intervention programs. Journal of Educational Psychology, 92, 284-295.

Schöler, H., \& Roos, J. (2010). Ergebnisse einer Evaluation von Sprachfördermaßnahmen in Mannheimer und Heidelberger Kitas. In K. Fröhlich-Gildhoff, I. Nentwig-Gesemann, \& P. Strehmel (Hrsg.), Forschung in der Frühpädagogik III, Schwerpunkt: Sprachentwicklung \& Sprachförderung: Bd. 5. Materialien zur Frühpädagogik (S. 35-74). Freiburg: FEL.

Schrey-Dern, D. (2006). Sprachfördermaßnahmen in Deutschland: Chancen und Risiken für die Berufsgruppe der Logopäden. Forum Logopädie, 5(20), 12-16. 
Senatorin für Soziales, Jugend, Frauen, Integration und Sport, Bremen. (2012). Rahmenplan für Bildung und Erziehung im Elementarbereich. Frühkindliche Bildung in Bremen. 2. Aufl. - https://www.soziales.bremen.de/jugend/detail. php?template $=20 \_$search_d\&search $\% 5$ Bsend $\% 5$ $\mathrm{D}=$ true \&search $\% 5 \mathrm{Bvt} \% 5 \mathrm{D}=$ rahmenplan $+\mathrm{f} \% \mathrm{FC}$ $\mathrm{r}+$ bildung+und+erziehung\&lang $=$ de \&skip $=30$. Zugegriffen: 6. Mai 2018.

Simon, S., \& Sachse, S. (2011). Sprachförderung in der Kindertagesstätte - Verbessert ein Interaktionstraining das sprachförderliche Verhalten von Erzieherinnen? Empirische Pädagogik, 25(4), $462-480$.

Simon, S., \& Sachse, S. (2013). Anregung der Sprachentwicklung durch ein Interaktionstraining für Erzieherinnen. Diskurs Kindheits- und Jugendforschung, 4, 379-397.

Strehmel, P. (2010). Einführungsbeitrag: Sprachförderung in Kindertagesstätten - Theorien, empirische Befunde, Anforderungen an die Praxis. In K. Fröhlich-Gildhoff, I. Nentwig-Gesemann, \& P. Strehmel (Hrsg.), Forschung in der Frühpädagogik III, Schwerpunkt: Sprachentwicklung \& Sprachförderung: Bd. 5. Materialien zur Frühpädagogik. Freiburg: FEL.

Tietze, W., Roßbach, H. G., \& Grenner, K. (2005). Kinder von 4 bis 8 Jahren. Zur Qualität der
Erziehung und Bildung in Kindergarten, Grundschule und Familie. Weinheim: Beltz.

Tracy, R. (2003). Sprachliche Frühförderung - Konzeptuelle Grundlagen eines Programms zur Förderung von Deutsch als Zweitsprache im Vorschulalter. Mannheim: Universität Mannheim, Forschungs- und Kontaktstelle Mehrsprachigkeit.

Tracy, R., \& Lehmke, V. (2009). Sprache macht stark. Berlin: Cornelsen.

Ulich, M., \& Mayr, T. (2003). SISMIK. Sprachverhalten und Interesse an Sprache bei Migrantenkindern in Kindertageseinrichtungen (Beobachtungsbogen und Begleitheft). Freiburg: Herder.

Ulich, M., \& Mayr, T. (2006). SELDAK. Sprachentwicklung und Literacy bei deutschsprachig aufwachsenden Kindern (Beobachtungsbogen und Begleitheft). Freiburg: Herder.

Weber, J., Marx, P., \& Schneider, W. (2007). Die Prävention von Lese- und Rechtschreibschwierigkeiten bei Kindern nicht-deutscher Herkunftssprache durch ein Training der phonologischen Bewusstheit. Zeitschrift für Pädagogische Psychologie, 21, 65-75.

Winner, A. (2007). Kleinkinder ergreifen das Wort. Sprachförderung von Kindern von null bis vier Jahren. Berlin: Cornelsen. 